\title{
Evaluation of the solitary pulmonary nodule by positron emission tomography imaging
}

\author{
T. Bury*, A. Dowlati*, P. Paulus**, J.L. Corhay*, T. Benoit**, J.M. Kayembe*, \\ R. Limet+, P. Rigo**, M. Radermecker*
}

Evaluation of the solitary pulmonary nodule by positron emission tomography imaging. T. Bury, A. Dowlati, P. Paulus, J.L. Corhay, T. Benoit, J.M. Kayembe, R. Limet, P. Rigo, M. Radermecker. CERS Journals Ltd 1996.

ABSTRACT: Current noninvasive imaging methods are not sufficiently reliable for accurate detection of malignancy in most solitary pulmonary nodules (SPNs). Positron emission tomography (PET) using 18-fluorodeoxyglucose (FDG), showing increased FDG uptake and retention in malignant cells, has proved useful to differentiate malignant from benign tissue and could, therefore, contribute to the evaluation of the SPN.

We performed a prospective study of 50 patients referred to the Pneumology Department with unclear diagnoses of SPN after conventional radiological screening. PET study was performed on each subject before an invasive procedure was proposed.

Thirty three patients had a malignant nodule and 17 had a benign nodule. The mean size of malignant nodule was $3 \mathrm{~cm}$ (range $1.5-4.5 \mathrm{~cm})$. All showed a marked increase in 18-FDG uptake. The mean size of benign nodule was $1.8 \mathrm{~cm}$ (range $0.5-3.5 \mathrm{~cm})$. PET imaging showed the absence of 18-FDG uptake and correctly identified 15 of 17 benign nodules. There were two false-positive cases with a moderate increase in 18-FDG uptake (1 postprimary tuberculosis; and 1 anthracosilicotic nodule with nonspecific inflammation). At present, the sensitivity and specificity of the method are 100 and $88 \%$, respectively. The positive and negative predictive values of PET imaging for SPNs are 94 and $100 \%$, respectively.

Our preliminary results demonstrate that PET-FDG imaging is a noninvasive technique, which appears highly accurate in differentiating malignant SPN from benign SPN.

Eur Respir J., 1996, 9, 410-414.
Depts of *Pneumology, **Nuclear Medicine, and +Thoracic Surgery, CHU Liège, Belgium.

Correspondence: Th. Bury

Pneumology Dept

CHU Sart Tilman B35

4000 Liège

Belgium

Keywords: Fluorodeoxyglucose positron emission tomography pulmonary nodule

Received: July 131995

Accepted after revision December 41995
The solitary pulmonary nodule (SPN) frequently presents a diagnostic dilemma (benign or malignant lesion?) to the chest physician. A SPN is defined by its radiographic appearance. The nodule must be solitary, surrounded by normal lung tissue, and generally not greater than $4.0 \mathrm{~cm}$ in its largest diameter $[1,2]$. By definition, patients with radiographic evidence of hilar or mediastinal adenopathy are excluded. The goal of radiological evaluation of a SPN, of course, is to distinguish between benign and malignant disease. The investigative approach often requires different techniques. The current noninvasive methods for evaluating the SPN include chest radiography, computed tomography (CT) and magnetic resonance imaging. Despite advances in morphological imaging and the realization of invasive procedures (bronchoscopy, percutaneous lung biopsy), the number of benign nodules which are resected remains $20-40 \%$ [1, 3-5].

Malignant tumours are generally characterized by uncontrolled cellular proliferation, with subsequent increased metabolic activity $[6,7]$. This increase of metabolic activity, both for glucose and amino acids, may be assessed by positron emission tomography (PET) [8]. In our centre, fluorine-18-deoxyglucose (18-FDG) is used as a routine radiopharmaceutical tracer to evaluate the increased glucose metabolism in tumour cells, which have a high rate of glucose consumption. A prospective study was performed in 50 patients to determine the diagnostic value of 18-FDG-PET in the aetiological diagnosis of SPN.

\section{Patients and methods}

\section{Patient selection}

Fifty subjects (mean age 64 yrs; range 19-81 yrs) who presented the evaluation of a SPN, were enrolled in this study. They were evaluated at the Pneumology Department of Liège University.

Each subject had chest radiographs and a CT scan (PQ 2000 - fourth generation; Picker, Cleveland, OH, USA) 
of the chest. All SPNs were noncalcified and no specific diagnosis could be achieved on the basis of morphological imaging. PET study was performed on each subject before an invasive procedure was proposed. Specimens for pathological evaluation were obtained by means of transbronchial or bronchial biopsy $(n=17)$, guided percutaneous needle aspirate $(n=11)$, or thoracotomy $(n=22)$. The protocol of this study was approved by the Hospital Ethics Committee.

\section{PET study}

Prior to the PET study, all patients fasted for $6 \mathrm{~h}$. PET was performed using a UGM Penn PET $240 \mathrm{H}$ scanner. This machine consists of six large areas $(50 \times 20 \mathrm{~cm}) \mathrm{Nal}$ crystals of $2.5 \mathrm{~cm}$ thickness arranged in an hexagonal array. The system has no septa or shadow shield but operates in 3D with an acceptance angle of 6.5 degrees. Opposed crystals are separated by $80 \mathrm{~cm}$ but the effective field of view is $512 \mathrm{~mm}$ in the $\mathrm{X}$ and $\mathrm{Y}$ directions and $128 \mathrm{~mm}$ in the $\mathrm{Z}$ direction. Images are reconstructed as 32 transverse planes composed of $4 \mathrm{~mm}$ isotropic voxels to form a $128 \times 128$ matrix. It is also possible to reconstruct 64 transverse planes using $2 \mathrm{~mm}$ voxels but with a field of view limited to $256 \mathrm{~mm}$.

The whole body mode acquires multiple overlapping steps at $6.4 \mathrm{~mm}$ intervals. This acquisition sequence corrects for the uneven interplane sensitivity inherent to 3D data acquisition schemes (diminished acceptance angle at the edges). Images are reconstructed using a Hanning filter. The effective spatial resolution of clinical data is $\approx 8 \mathrm{~mm}$ (full width at half maximum (FWHM)), whilst the intrinsic resolution of the machine using phantoms and a ramp filter is $5.5 \mathrm{~mm}$ (specification). Six to 10 steps $(512-768 \mathrm{~mm})$ extending from the neck to the abdomen total scanning time 40-60 $\mathrm{min}\left(4-8 \mathrm{~min} \cdot \mathrm{step}^{-1}\right)$, are acquired for each patient.

PET data were analysed by visual interpretation of coronal, sagital and transverse slices alone and in crossreferenced situation. This method of analysis has been validated by comparison to quantitative data $[9,10]$.

PET images were read independently by two groups of two nuclear physicians, who had knowledge of the standard chest radiographs only. They evaluated the presence or the absence of 18-FDG uptake in the SPN. When an increase in 18-FDG uptake was observed, two levels were identified: moderate or intense. At the end of the first step of interpretation, the two teams of reviewers compared their findings. When they did not agree, the teams reviewed the images together to reach a consensus. In this study, there was one instance of disagreement about PET images.

\section{Statistical analysis}

Diagnostic efficacy of PET-FDG imaging to differentiate malignant from benign SPN was evaluated by calculating sensitivity, specificity, positive predictive value and negative predictive value. For each parameter, the $95 \%$ confidence intervals $(95 \% \mathrm{CI})$ was given [11].
Table 1. - Clinical data in 50 patients with SPN

\begin{tabular}{lccc}
\hline & Malignant & Benign & Total \\
\hline Patients n & 33 & 17 & 50 \\
Sex ratio M/F & $29 / 4$ & $8 / 9$ & $37 / 13$ \\
$\begin{array}{l}\text { Mean age yrs } \\
\text { Nodule size cm }\end{array} \quad 64$ & 64 & 64 \\
$\quad 1.5$ & 1 & 7 & 8 \\
$\quad 1.6-3.5$ & 23 & 10 & 33 \\
$>3.5$ & 9 & 0 & 9 \\
\hline
\end{tabular}

M: male; F: female; SPN: solitary pulmonary nodule.

\section{Results}

Clinical data from the 50 patients are listed in table 1. Thirty three patients had a malignant nodule and 17 had a benign nodule.

For malignant nodules, histological diagnosis was confirmed by analysis of fragments obtained by bronchoscopy $(n=14)$, transthoracic fine needle aspiration biopsy $(n=7)$, or thoracotomy $(n=12)$. The histological diagnoses in the 33 patients with malignant SPN included adenocarcinoma $(n=11)$; squamous cell carcinoma $(n=18)$; small cell carcinoma $(n=3)$; and malignant carcinoid tumour $(n=1)$. The mean size of these malignant nodules was $3 \mathrm{~cm}$ (range $1.5-4.5 \mathrm{~cm}$ ).

Seventeen patients had a benign disease. The disease was considered benign only after the performance of a general and complete thoracic check-up: 2 chronic abscesses, 4 sclerous nodules (nonspecific inflammatory processes); 4 hamartomas; 2 anthracosilicotic nodules; 2 nonactive tuberculomas; 1 active tuberculosis; 2 pneumonia sequelae (table 2). The mean size of benign nodules was $1.8 \mathrm{~cm}$ (range $0.5-3.5 \mathrm{~cm}$ ). This diagnosis was supported either by the absence of malignant cells in histological specimens obtained by invasive procedures (bronchial or transbronchial biopsies $n=3$; transthoracic needle biopsies $n=4$; thoracotomies $n=10$ ), by the disappearance of infectious processes after adapted chemotherapy $(n=2)$, or by the chest radiological stability of the nodule over 2.5 yrs $(n=1)$.

The clinical evolution under antimicrobial chemotherapy confirmed the diagnosis of chronic abscess $(n=2)$. Ten of the other 15 subjects with benign lesions had the histological diagnosis established by means of thoracotomy. Another patient had a nonactive tuberculoma: the diagnosis was supported by the clinical presentation and backed by transthoracic needle aspiration showing necrotizing granuloma. Transthoracic needle aspiration biopsies had been performed in two patients under CT guidance and in communication and co-operation with an experienced pathologist; the histological diagnosis in both patients was a hamartoma $(n=2)$. One patient underwent invasive exploration (bronchoscopic and transthoracic biopsies) on two occasions at a $1 \mathrm{yr}$ interval and was diagnosed as having a sclerous nodule (nonspecific inflammation). This nodule appeared stable at 2.5 year follow-up. The last patient with a benign nodule had an active post primary tuberculosis. 
Table 2. - Clinical and histological findings in 17 patients with benign pulmonary nodule

\begin{tabular}{cccclc}
\hline $\begin{array}{l}\text { Pt } \\
\text { No. }\end{array}$ & $\begin{array}{c}\text { Age } \\
\text { yrs }\end{array}$ & Sex & $\begin{array}{c}\text { Nodule size } \\
\text { cm }\end{array}$ & \multicolumn{1}{c}{ Diagnostic } & $\begin{array}{c}\text { Biopsy } \\
\text { mode }\end{array}$ \\
\hline 1 & 67 & F & 2.0 & Abscess & Bronchoscopy \\
2 & 81 & M & 1.5 & Sclerous nodule & Thoracotomy \\
3 & 58 & F & 3.0 & Hamartoma & Transthoracic \\
4 & 61 & M & 2.0 & Abscess & Bronchoscopy \\
5 & 68 & M & 2.0 & Anthracosilicotic nodule & Thoracotomy \\
6 & 51 & F & 3.5 & Tuberculoma & Thoracotomy \\
7 & 51 & F & 1.5 & Sclerous nodule & Thoracotomy \\
8 & 74 & M & 2.0 & Sclerous nodule & Transthoracic \\
9 & 61 & F & 2.0 & Tuberculoma & Transthoracic \\
10 & 57 & F & 1.4 & Hamartoma & Thoracotomy \\
11 & 64 & M & 2.5 & Anthrocosilicotic nodule & Thoracotomy \\
12 & 55 & F & 0.5 & Hamartoma & Thoracotomy \\
13 & 75 & M & 2.3 & Active tuberculosis & Bronchoscopy \\
14 & 52 & F & 0.8 & Sclerous nodule & Thoracotomy \\
15 & 74 & F & 1.8 & Organizing Pneumonia & Thoracotomy \\
16 & 69 & M & 1.4 & Hamartoma & Transthoracic \\
17 & 68 & M & 1.3 & Organizing pneumonia & Thoracotomy \\
\hline
\end{tabular}

Pt: patient; M: male; F: female.

\section{PET imaging findings}

PET images were analysed by visual interpretation. Results are shown in table 3. PET imaging correctly identified 48 of the 50 SPN. The present sensitivity of the method is $100 \%$ (95\% CI $89.4-100 \%)$, with a negative predictive value of $100 \%$ (95\% CI $78.2-100 \%)$. All malignant nodules showed an increase in 18-FDG uptake, which was intense in 32 cases and moderate in 1 case (well-differentiated squamous cell carcinoma). Among patients with malignant nodules, one patient was surgically treated for bronchial carcinoid tumour. Histologically, this tumour was classified as an atypical carcinoid (classification of ARRIGONI et al. [12]). As mentioned above, the other histological lesions were: adenocarcinoma $(n=11$, of which one was of the bronchioloalveolar type); squamous cell carcinoma $(n=18)$; and small cell carcinoma $(n=3)$. Figure 1 shows CT and PET-FDG scans in a 75 year old man with a squamous cell carcinoma with $1.8 \times 1.7 \mathrm{~cm}$ nodule in the left lower lobe, note the intense FDG uptake in the nodule.

PET imaging showed the absence of 18-FDG uptake and correctly identified 15 of 17 benign nodules: 2 chronic abscesses; 4 sclerous nodules; 4 hamartomas; 1 anthracosilicotic nodule; 2 nonactive tuberculomas; 2 pneumonia sequelae.

Two benign nodules showed an increase in FDG uptake, which was moderate. One patient had a history

Table 3. - PET imaging results: correlation with clinical diagnosis of disease

\begin{tabular}{lcc}
\hline & \multicolumn{2}{c}{ Clinical diagnosis of disease } \\
\cline { 2 - 3 } FDG-PET & Benign & Malignant \\
\hline Negative & 15 & 0 \\
Positive & 2 & 33
\end{tabular}

FDG: 18-fluorodeoxyglucose; PET: positron electron tomography. compatible with a postprimary tuberculosis and bronchial secretion aspirations were positive for acid fast bacilli at traditional culture. The second patient had a thoracotomy with the histological diagnosis of anthracosilicotic nodule associated with a chronic nonspecific

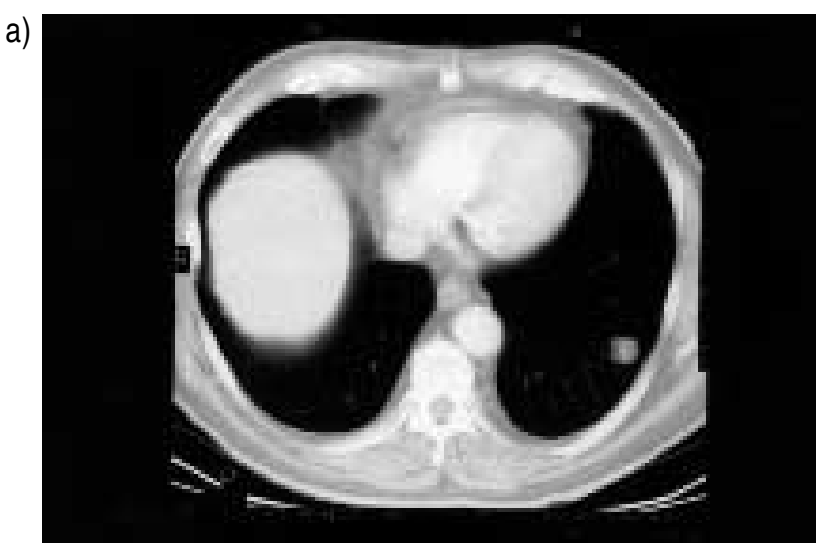

b)

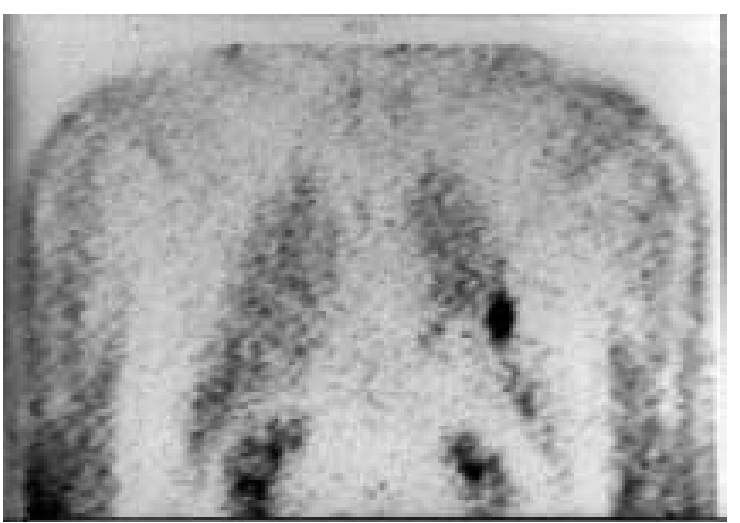

Fig. 1. - a) Computed tomography (CT) scan; and b) PET-FDG in a $75 \mathrm{yr}$ old man with squamous cell carcinoma of the left lower lobe. Note the intense FDG uptake in the nodule. PET: positron emission tomography; FDG: 18-fluorodeoxyglucose. 
inflammation. At present, the specificity of the FDGPET imaging is $88 \%$, with $95 \%$ CI of $63.3-98.5 \%$. In our series, the positive predictive value is $94 \%$ (95\% CI 80.8-99.3\%).

Presence of distant metastatic lesions $(n=5)$ was confirmed by biopsy in four patients: 3 hepatic lesions; 1 adrenal lesion; and 1 lymph node involvement. All these lesions showed an intense FDG uptake by PET imaging.

\section{Discussion}

Our study shows that PET-FDG imaging is a useful noninvasive technique for accurate differentiation of benign and malignant SPNs. Currently, approximately $40 \%$ of SPNs are malignant, either bronchogenic carcinoma or metastatic from an extrathoracic site [4, 13]. This percentage can significantly vary depending on age, smoking habits and prevalence of other disease in the country. For example, lung carcinoma is extremely rare in patients less than $30 \mathrm{yrs}$ of age whereas in patients aged more than 50 yrs, over one half of SPNs are malignant lesions. In our study, the mean age of patients was 64 yrs. This probably explains the $66 \%$ malignancy rate among the SPNs observed in our series.

The classical management of the SPN is prompt surgical removal, unless a benign condition can be demonstrated. Computed tomography (CT) and magnetic resonance imaging provide anatomical and morphological information concerning the lesions. Two radiological criteria suggest the benign condition of a nodule: the presence of central, concentric calcification on chest radiographs or CT scan and the stability of the nodule for more than 2 yrs [1, 14]. Other criteria for benign disease include a transthoracic needle aspiration biopsy demonstrating a specific benign diagnosis, and patient age less than 30-35 yrs, unless a prior extrapulmonary malignancy exists [15]. Despite advances in morphological imaging, the number of resected nodules which are benign remains $20-40 \%$. Thus, a substantial number of patients (e.g., elderly patients or those with poor cardiopulmonary condition) are exposed to postoperative complications [1, 3-5].

PET-FDG imaging is a noninvasive technique which depends mainly on the metabolic characteristics of a tissue for the diagnosis of disease. Indeed, malignant tumour cells have been found to have a high rate of glycolysis and to be associated with increased membrane glucose transfer capability $[6,7]$. FDG has proved useful for tracing glucose metabolism, detecting malignant tissue and quantifying changes in tumour glycolysis during therapeutic management $[4,16]$. In the past $5 \mathrm{yrs}$, an increasing number of applications of PET have been introduced for diagnosing and staging various types of cancers, particularly in colorectal, oropharynx and brain tumours [8, 17-19]. The applications of PET in thoracic oncology have not yet been extensively studied. It appears that this technique is very useful in lung tumour staging and also in the evaluation of the success of chemotherapy [9, 20-22]. In our study, PET imaging correctly identified
48 of the 50 solitary pulmonary nodules. At present, this technique has a sensitivity of $100 \%$. All malignant SPN have shown an increase in 18-FDG uptake, which was intense in 32 cases and moderate in 1 case.

The moderate 18-FDG uptake in malignant SPN was seen in one patient with an adenocarcinoma of $2.6 \mathrm{~cm}$. A review of the chest radiographs showed that a $2 \mathrm{~cm}$ nodule was already present 2 yrs earlier, suggesting slow growth of the tumour. Indeed, the detectability of a lesion with PET scan depends not only on the size of the lesion but also on the radiotracer concentration. It is likely that a critical mass of metabolically active malignant cells is required for PET diagnosis. In our series of malignant nodules, we did not observe any difference in FDG uptake, whatever the histopathological type of the malignant nodule; small cell carcinoma, squamous cell carcinoma, adenocarcinoma and even carcinoid tumour showed a similar intense FDG uptake. This is not in concordance with the notion that tumour doubling time is generally related to the histological type of the tumour [23, 24]. However, we have little information on the growth rate of nodules in our patients. It is also possible that a quantitative analysis of PET imaging would be necessary to clarify this point.

PET imaging correctly identified 15 of the 17 benign nodules. The aetiological diagnoses were accepted after exhaustive exploration (clinical, bacteriological and imaging data; histological specimens): 2 chronic abscesses; 2 nonactive tuberculomas; 4 harmartomas; 4 sclerous nodules (nonspecific inflammation); 1 anthracosilicotic nodule; 2 pneumonia sequelae. On the other hand, two false-positive results were seen, one in a patient with a $2 \mathrm{~cm}$ anthracosilicotic nodule and the second in a patient with caseating granulomas and active inflammation due to tuberculosis. In these two cases, 18-FDG uptake in the nodule was moderate. At present, the specificity of the technique is $88 \%$, with $95 \%$ CI of $63.8-98.5 \%$.

It is clear that FDG uptake is not specific for malignant tissue and it has been reported in other conditions, such as bacterial pneumoniae, acute pyogenic abscesses, active sarcoidosis, cryptogenetic fibrosing alveolitis and infectious granulomas [25-28]. For this reason, the specificity and positive predictive value of PET imaging may vary among different areas of the world depending on the prevalence of granulomatous diseases. In these pulmonary lesions, the FDG uptake has been attributed to an increase in macrophage and granulocytic activity. Our study is in concordance with three earlier reports which showed that PET-FDG imaging was more precise than CT in the evaluation of SPNs [21, 29, 30]. These studies showed that PET-FDG imaging is very useful in detecting malignancy in SPNs with a sensitivity varying between 89-95\%. In the study of DewAN et al. [29], there was one false-negative result in a patient with a 1 $\mathrm{cm}$ nodule identified as a scar adenocarcinoma.

In summary, PET-FDG imaging is a noninvasive technique, which appears highly accurate in differentiating malignant SPN from benign SPN. The validation of this technique in larger series of patients will probably allow a reduction in the number of invasive diagnostic procedures or thoracotomies in the management of SPNs. 


\section{References}

1. Swensen SJ, Jett JR, Payne WS, Viggiano RW, Pairolero PC, Trastek VF. An integrated approach to evaluation of the solitary pulmonary nodule. Mayo Clin Proc 1990; 65: 173-186.

2. Walske BR. The solitary pulmonary nodule: a review of 217 cases. Dis Chest 1966; 49: 302-304.

3. Khouri NF, Meziane MA, Zerhouni EA, Fishman EK, Siegelman SS. The solitary pulmonary nodule, assessment, diagnosis and management. Chest 1987; 91: 128-133.

4. Lillington GA. Pulmonary nodules: solitary and multiple. Clin Chest Med 1982; 3: 361-367.

5. Zerhouni EA, Stitik FP, Siegelman SS, et al. CT of the pulmonary nodules: a co-operative study. Radiology 1986; 160: 319-327.

6. Hatamaka M. Transport of sugars in tumor cell membranes. Biochem Biophys Acta 1974; 355: 74-104.

7. Flier JS, Mueckleer MM, Usher P, Lodish HF. Elevated levels of glucose transport and transporter messenger RNA are induced by ras and sarc oncogenes. Science 1987; 23: 1492-1495.

8. Strauss LG, Conti PS. The applications of PET in clinical oncology. J Nucl Med 1991; 32: 623-648.

9. Patz E, Lowe V, Hoffman J, Paine S, Harris L, Goodman $P$. Persistent or recurrent bronchogenic carcinoma: detection with PET and 2-[F-18]-2-deoxy-D-glucose. Radiology 1994; 191: 379-382.

10. Lowe VJ, Hoffman JM, Delong DM, Patz EF, Coleman RE. Semiquantitative and visual analysis of FDG-PET images in pulmonary abnormalities. J Nucl Med 1994; 35: 1771-1776.

11. Diem K. Tables scientifiques. Documenta Geigy. 6ème édition. J.R. Geigy SA, ed. Basle, 1963; pp. 85-103.

12. Arrigoni MG, Woolner LB, Bernatz PE. Atypical carcinoid tumors of the lung. J Thorac Cardiovasc Surg 1972; 64: 413-421.

13. Midthun DE, Swensen SJ, Jett JR. Clinical strategies of solitary pulmonary nodule. Ann Rev Med 1992; 43: 195-208.

14. Zerhouni EA, Khouri NF, Leo FP, Fishman EK, Braverman RM. Solitary pulmonary nodules: CT assessment. Radiology 1986; 160: 307-312.

15. Mack MJ, Hazelrigg SR, Landreneau RJ, Acuff TE. Thoracoscopy for the diagnosis of the indeterminate solitary pulmonary nodule. Ann Thorac Surg 1993; 56: 825-832.

16. Hoh C, Hawkins R, Glaspy J, et al. Cancer detection with whole-body PET using 2-[18F]fluoro-2-deoxy-Dglucose. J Comput Assist Tomogr 1993; 17: 582-589.
17. Knopp MV, Strauss LG, Haberkorn U, et al. PET for the diagnostic work up in thoracic oncology. Eur J Nucl Med 1990; 16: 560 (Abstract).

18. Minn H, Joensuu H, Ahonen A, Klesni P. Fluorodeoxyglucose imaging: a method to assess the proliferative activity of human cancer in vivo. Comparison with DNA flow cytometry in head and neck tumors. Cancer 1988; 61: 1776-1781.

19. Alavi JB, Alavi A, Chawluk J. Positron emission tomography in patients with glioma: a prediction of prognosis. Cancer 1988; 62: 1074-1078.

20. Lewis P, Griffin S, Marsden P, et al. Whole-body 18F-fluorodeoxyglucose positron emission tomography in preoperative evaluation of lung cancer. Lancet 1994; 344: $1265-1266$.

21. Gupta N, Franck A, Dewan N, et al. Solitary nodules: detection of malignancy with PET with 2-[18F]-fluoro2-deoxy-D-glucose. Radiology 1992; 184: 441-444.

22. Whal R, Quint L, Greenough R, Meyer C, White R, Orringer M. Staging of mediastinal non-small cell lung cancer with FDG PET, CT and fusion images: preliminary prospective evaluation. Radiology 1994; 191: 371377.

23. Collins VP, Loeffler RK, Tivey H. Observations on growth rates of human tumors. Am J Roentgenol Rad Ther 1956; 76: 988-1000.

24. Kerr KM, Lamb D. Actual growth rate and tumour cell proliferation in human pulmonary neoplasms. Br J Cancer 1984; 50: 343-349.

25. Brudin L, Valind S, Rhodes C, et al. Fluorine-18 deoxyglucose uptake in sarcoidosis measured with positron emission tomography. Eur J Nucl Med 1994; 21: $297-$ 305.

26. Pantin C, Valind S, Sweatman M, et al. Measures of the inflammatory response in cryptogenic fibrosing alveolitis. Am Rev Respir Dis 1988; 138: 1234-1241.

27. Haslett C, Clark R, Jones H, Krausz T, Rhodes C. Neutrophil metabolic activity in localized pulmonary inflammation measured non-invasively by positron emission tomography (PET). Physiologist 1989; 32: 210 213.

28. Kubota K, Matsuzawa T, Fujiwara T, et al. Differential diagnosis of lung tumor with positron emission tomography a prospective study. J Nucl Med 1990; 31: 1927 1933.

29. Dewan N, Gupta N, Redepenning L, Phallen J, Frick M. Diagnostic efficacy of PET-FDG imaging in solitary pulmonary nodules. Chest 1993; 104: 997-1002.

30. Patz E, Lowe V, Hoffman J, et al. Focal pulmonary abnormalities: evaluation with F-18 fluorodeoxyglucose PET scanning. Radiology 1993; 188: 487-490. 